\title{
An In Vitro Investigation of Anticaries Efficacy of Fluoride Varnishes
}

\author{
L Al Dehailan • EA Martinez-Mier • GJ Eckert• F Lippert
}

\begin{abstract}
Clinical Relevance
This study provides clinicians with insight into the in vitro differences in the performance of commercially available fluoride varnishes. More clinical research is needed to equip dentists with evidence that will help them make better treatment choices.
\end{abstract}

\section{SUMMARY}

Most currently marketed fluoride varnishes (FVs) have not been evaluated for their effectiveness in preventing dental caries. The objective of this study was to investigate the anticaries efficacy, measured as fluoride release into artificial saliva (AS); change in surface microhardness of early enamel caries lesions; and enamel fluoride uptake (EFU) of 14 commercially available FVs and two control groups. Bovine enamel specimens $(5 \times 5 \mathrm{~mm})$

*Laila Al Dehailan, Department of Restorative Dental Sciences, College of Dentistry, Imam Abdulrahman Bin Faisal University, Dammam, KSA, and Department of Cariology, Operative Dentistry and Dental Public Health, Indiana University School of Dentistry, Indianapolis, IN, USA

Esperanza Angeles Martinez-Mier, Department of Cariology, Operative Dentistry and Dental Public Health, Indiana University School of Dentistry, Indianapolis, IN, USA

George J Eckert, Department of Biostatistics, School of Medicine, Indiana University, Indianapolis, IN, USA

Frank Lippert, Department of Cariology, Operative Dentistry and Dental Public Health, Indiana University School of Dentistry, Indianapolis, IN, USA

*Corresponding author: PO Box 1982, Dammam 31441, Kingdom of Saudi Arabia; e-mail: ldehailan@iau.edu.sa

DOI: https://doi.org/10.2341/18-040-L were prepared and assigned to 18 groups $(n=12)$. Early caries lesions were created in the specimens and characterized using Vickers microhardness $\left(\mathrm{VHN}_{\text {lesion }}\right)$. FV was applied to each group of specimens. Immediately afterward, specimens were incubated in $4 \mathrm{~mL}$ of AS for 18 hours, which were collected and renewed every hour for the first six hours. AS samples were analyzed for fluoride using an ion-specific electrode. Specimens were then brushed for 20 seconds with toothpaste slurry and subjected to $\mathrm{pH}$ cycling consisting of a four-hour/day acid challenge and one-minute treatments with 1100 ppm $F$ dentifrice for five days. Microhardness was measured following pH cycling $\left(\mathrm{VHN}_{\text {post }}\right)$. EFU was determined using microbiopsy. Acid resistance (eight-hour demin challenge) was performed after pH cycling, and microhardness was measured $\left(\mathrm{VHN}_{\text {art }}\right)$ and compared with baseline values to test the FV impact after pH cycling. One-way analysis of variance was used for data analysis $(\alpha=0.05)$. FVs differed in their release characteristics (mean \pm SD ranged from $14.97 \pm 2.38$ $\mu \mathrm{g} / \mathrm{mL}$ to $0.50 \pm 0.15 \mu \mathrm{g} / \mathrm{mL}$ ), rehardening capability (mean \pm SD ranged from $24.3 \pm 15.1$ to $11.7 \pm 12.7)$, and ability to deliver fluoride to demineralized lesions (mean \pm SD ranged from

\footnotetext{
"Used by permission. () Operative Dentistry, Inc. Transmission or reproduction of protected items beyond that allowed by fair use requires the written permission of Operative Dentistry, Inc."
} 
$3303 \pm 789 \mu \mathrm{g} / \mathrm{cm}^{3}$ to $\left.707 \pm 238 \mu \mathrm{g} / \mathrm{cm}^{3}\right)$. Statistically significant but weak linear associations were found between $\Delta \mathrm{VHN}_{\text {(post - lesion), }}$ EFU, and fluoride release (correlations 0.21-0.36). The results of this study demonstrated that differences in FV composition can affect their efficacy in in vitro conditions.

\section{INTRODUCTION}

The effects of topically applied fluoride treatments have been well known since the 1930s and have resulted in beneficial outcomes in caries reduction. ${ }^{1-5}$ Several studies have confirmed the effectiveness of fluoride varnishes (FVs) in reducing caries incidence and prevalence with the added benefits of ease of use and, most importantly, virtually no side effects. ${ }^{6-8}$

In vitro models are very commonly used in cariology research. Most established models are designed to mimic clinical conditions as closely as possible. In vitro models have controlled scientific settings that limit variability and increase sensitivity compared with clinical models. However, they come with their own limitations in their inability to fully simulate the complex intraoral environment, especially circumstances leading to formation of dental caries. ${ }^{9-11}$

Most of the available in vitro studies that investigate the efficacy of FVs vary in their experimental design and testing conditions. ${ }^{12,13}$ This creates heterogeneity, making it difficult to find benchmark tests that accurately assess efficacy. Therefore, we have selected to investigate different research variables that are of importance when studying fluoride efficacy in an attempt to improve our understanding of FVs and find the best test conditions. We also tried to study how those variables relate to each other in an effort to establish relevant variables for in vitro FV research.

The aim of our study was to investigate the potential anticaries effect of 14 commercially available FVs on artificially created early caries lesions through the following outcome variables: 1) 24-hour fluoride release into artificial saliva (AS), b) surface microhardness, and c) enamel fluoride uptake (EFU), after $\mathrm{pH}$ cycling for five days with a second demineralization challenge.

\section{METHODS AND MATERIALS}

\section{Specimen Preparation}

Enamel specimens, $5 \times 5 \mathrm{~mm}$, were obtained from bovine teeth using a low-speed saw (IsoMet, Buehler, Lake Bluff, IL, USA). The teeth were stored in deionized water saturated with thymol during the sample preparation process. The specimens were ground and polished to create flat surfaces to facilitate surface microhardness testing using the Struers RotoPol 31/RotoForce 4 polishing unit (Struers Inc, Cleveland, PA, USA). The bottom side of the specimens was ground flat to a uniformthickness using 500-grit silicon carbide grinding paper. The topside of the specimens was ground in a series of 1200-, 2400-, and 4000-grit paper until most of the tooth surface was flattened. The specimens were then polished using $1-\mu \mathrm{m}$ diamond paste to eliminate any scratches that may have resulted from the grinding procedure. The specimens were sonicated in deionized water between each grinding/polishing step. As a final cleaning step, the polished specimens were sonicated in $2 \%$ microliquid. The specimens were assessed with a magnification of $10 \times$.

To be acceptable for the study, a specimen was required to

1) have a minimum $5 \times 5 \mathrm{~mm}$ polished facet across the surface;

2) not have any obvious cracks or other flaws in the enamel surface;

3) have an evenly polished, high-gloss enamel surface; and

4) have no contamination on the top surface from sticky wax or any other material.

Specimens were then secured, with the polished enamel side facing upward, on a one-inch square acrylic block using sticky wax to facilitate surface microhardness measurements. Then, all specimen surface areas apart from the polished top surface were covered with a colored nail varnish (Sally Hansen Advanced, Hard As Nails Nail Polish, Red, New York, NY, USA). Prepared specimens were stored at approximately $100 \%$ relative humidity and $4^{\circ} \mathrm{C}$ until further use. A total of 216 specimens were prepared for the present study (18 groups of 12 specimens each).

\section{Sound Enamel Microhardness}

Initial surface microhardness of the sound enamel specimens was determined using a Vickers microhardness indenter at a load of $200 \mathrm{~g}$ for 11 seconds (Instron T2100B Vickers Surface Microhardness Tester, Norwood, MA, USA). The average sound enamel microhardness $\left(\mathrm{VHN}_{\text {sound }}\right)$ was determined from five indentations on the surface of each specimen (Figure 1). Only specimens with $300 \leq$ $\mathrm{VHN}_{\text {sound }} \leq 400$ were accepted into the study. 


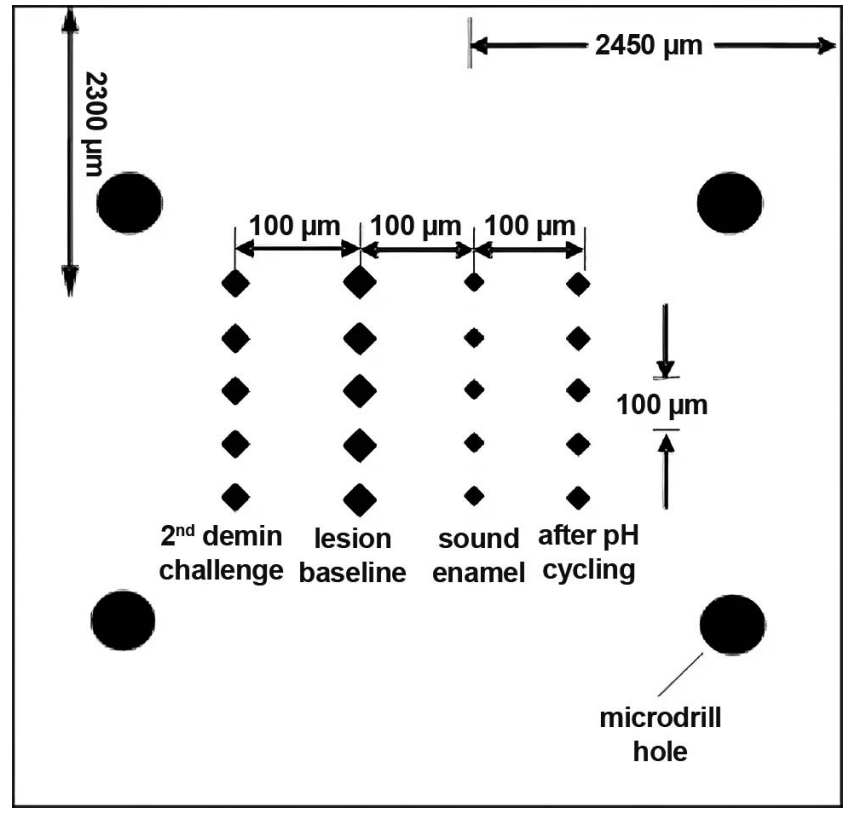

Figure 1. Enamel specimen $(5 \times 5 \mathrm{~mm})$ with microhardness indentations and microdrill holes.

\section{Artificial Lesion Creation}

Artificial lesions were formed in the enamel specimens by a 48 -hour immersion at $37^{\circ} \mathrm{C}$ into a solution of $0.1 \mathrm{M}$ lactic acid and $0.2 \%$ Carbopol C907, which was $50 \%$ saturated with hydroxyapatite and adjusted to $\mathrm{pH} 5.0$ using $\mathrm{KOH}$. After demineralization, the specimens were rinsed with deionized water for approximately one minute. Specimens were blotted dry with a tissue and stored at approximately $100 \%$ relative humidity and $4^{\circ} \mathrm{C}$ until further use.

\section{Lesion Baseline Microhardness}

Microhardness of the demineralized enamel specimens was determined as described above. The average specimen lesion baseline microhardness $\left(\mathrm{VHN}_{\text {lesion }}\right)$ was calculated. Only specimens with 25 $\leq \mathrm{VHN}_{\text {lesion }} \leq 60$ were accepted into the study. Specimens were assigned to treatment groups $(\mathrm{n}=12)$ based on a randomization procedure that resulted in treatment groups with mean $\mathrm{VHN}_{\text {lesion }}$ values that were not significantly different statistically.

\section{Specimen Mounting}

Once assigned to their treatment groups, specimens were removed from their acrylic blocks and mounted onto a 12-well microtiter plate lid. Acrylic blocks $(12 \times 12 \times 9 \mathrm{~mm})$ were mounted onto the inside of the lid using acrylic glue. Then, specimens were mounted with the enamel side facing upward onto the acrylic block using sticky wax.

\section{FV Application}

All products tested in this study contained $5 \% \mathrm{NaF}$ $(22,600 \mathrm{ppm}$ fluoride ion) and were assigned to groups and labeled as per Table 1 . In addition to the test FVs shown above, two additional experimental groups were included: one placebo group (O-; placebo varnish, no toothpaste treatment during $\mathrm{pH}$ cycling phase) and one positive control group $(\mathrm{O}+$; no FV treatment, toothpaste treatment during $\mathrm{pH}$ cycling phase). The placebo varnish had the following composition (all w/w): $2 \%$ shellac, $10 \%$ ethyl cellulose, $40 \%$ ethyl acetate, $2 \%$ polyvinylpyrrolidone, $2 \%$ xylitol, $5 \% \mathrm{NaCl}$, and $39 \%$ ethanol and was manufactured in-house especially for this study.

The protective foil from the individual FV dose was removed, and the FV was mixed using the manufacturer's applicator for at least 10 seconds to homogenize the $\mathrm{FV}$, as sedimentation of $\mathrm{NaF}$ and phase separation may have occurred during storage. Duraphat (group C) was supplied in a tube. For this $\mathrm{FV}$, approximately $0.5 \mathrm{~mL}$ was squeezed into a small weighing cup and processed as described above. The placebo varnish was handled in a similar manner.

Subsequently, FV was applied to the surface of each specimen using a single brush stroke and using the manufacturer's applicator. Any unused FV was discarded.

\section{Saliva Incubation}

Immediately after FV application, the lid containing 12 specimens was placed onto the microtiter plate containing $4.0 \mathrm{~mL}$ of AS per well. AS had the same composition as in a previous experiment. ${ }^{14}$ Two batches of this solution were prepared: one for the FV incubation phase in all specimens and one for the $\mathrm{pH}$ cycling phase for all specimens.

The microtiter plate was then placed in an incubator set at $37^{\circ} \mathrm{C}$. Every hour for six hours, the lid was placed onto a new microtiter plate containing $4.0 \mathrm{~mL}$ fresh AS per well.

This procedure was repeated until a total AS exposure time of six hours had been reached. After six hours, the lid was placed onto a new microtiter plate containing $4.0 \mathrm{~mL}$ AS per well, which was placed in the incubator for 18 hours. All saliva samples were frozen immediately after each cycle and retained for analysis of ionic fluoride. 


\begin{tabular}{|c|c|c|c|}
\hline $\mathrm{A}$ & CavityShield 5\% Varnish & 3M ESPE & - \\
\hline $\mathrm{C}$ & Colgate Duraphat Varnish & Colgate Oral Pharmaceuticals & - \\
\hline $\mathrm{D}$ & Colgate Prevident Varnish & Colgate Oral Pharmaceuticals & Xylitol \\
\hline $\mathrm{G}$ & Kolorz Clearshield Varnish & DMG America & Xylitol \\
\hline $\mathrm{H}$ & MI Varnish & GC America & Casein phosphopeptide-ACP (CPP-ACP, Recaldent) \\
\hline 1 & Duraflor Halo 5\% Sodium Fluoride Varnish & Medicom & - \\
\hline $\mathrm{J}$ & Enamel Pro Varnish Clear & Premier Dental & Amorphous calcium phosphate (ACP), Xylitol \\
\hline $\mathrm{K}$ & Vella Fluoride Varnish & Preventive Technologies & Xylitol \\
\hline $\mathrm{O}$ & Placebo Varnish & (manufactured in-house) & - \\
\hline
\end{tabular}

Then, a soft toothbrush (Oral B P40, Procter \& Gamble, Mason, OH, USA) was used to brush each specimen by hand. A slurry of Crest Cavity Protection $(0.243 \%$ sodium fluoride; Procter \& Gamble) at a ratio of $1: 2 \mathrm{w} / \mathrm{w}$ (dentifrice: AS) was prepared in a beaker with a magnetic stirrer. The toothbrush was dipped into the slurry briefly (approximately two seconds). Each specimen was then brushed under a stream of deionized water for 20 seconds, with the specimen being rinsed another 10 seconds after brushing. This procedure was repeated until all specimens on the lid were brushed. A new toothbrush and slurry was used for each lid. After this procedure, the $\mathrm{pH}$ cycling phase commenced on the same day.

\section{pH Cycling Phase: Remineralization}

The present study was separated into three phases, and each phase contained a placebo group (ie, $\mathrm{O}-\mathrm{a}$, $\mathrm{O}-\mathrm{b}, \mathrm{O}-\mathrm{c})$ to allow for comparisons between phases. The daily cyclic treatment regimen for each lid containing the specimens consisted of one, oneminute treatment period with a slurry of aforementioned toothpaste (prepared as described above), followed by a two-hour exposure to AS, four hours per day acid challenge in the lesion-forming solution, and exposure to AS for the remainder of the day and overnight. One slurry per day was prepared and pipetted into each well of the used microtiter plates.

The $\mathrm{pH}$ cycling was performed by placing the lid containing the specimens onto different microtiter plates containing $4.0 \mathrm{~mL}$ each per well of toothpaste slurry, AS, or lesion-forming solution. After each treatment, the specimens were rinsed under running deionized water briefly (approximately two seconds per specimen). The regimen was repeated for five days. The study was conducted at room temperature.

After completion of the $\mathrm{pH}$ cycling phase, all specimens were carefully removed from the lids and remounted onto an acrylic block to facilitate microhardness and EFU measurements.

\section{Posttreatment Microhardness}

The average specimen microhardness $\left(\mathrm{VHN}_{\text {post }}\right)$ was determined again in the same manner as when obtaining lesion and baseline microhardness.

\section{Enamel Fluoride Uptake}

The fluoride content of the enamel specimens was determined using the microdrill technique. The enamel specimens were mounted perpendicular to the long axis of a micro end mill attached to a specially designed microdrill and drilled to a depth of $100 \mu \mathrm{m}$ through the entire lesion. The drilling and sample collection were performed in a static-controlled atmosphere to prevent loss of enamel powder due to charging effects. The enamel powder sample was transferred to a diffusion dish and then analyzed for fluoride. The diameter of the drill hole was determined using a calibrated microscope interfaced with an image analysis system. Indentations for microhardness testing and microdrill holes were placed on enamel specimens according to Figure 1. 


\section{Acid Resistance Test}

To test whether the various FVs impart acid resistance to the enamel specimens after $\mathrm{pH}$ cycling, a second in vitro demineralization treatment was performed and followed the same protocol as described above but using a demineralization time of only eight hours. The average specimen microhardness $\left(\mathrm{VHN}_{\text {art }}\right)$ was determined again as described above.

\section{AS Fluoride Analysis}

The collected, frozen AS samples were thawed. An aliquot was removed and analyzed for fluoride by comparison to a similarly prepared standard curve using an ion-selective electrode (Model 9609BNWP, Orion Research, Boston, MA, USA) and meter. Individual as well as cumulative $\mathrm{F}$ release data were calculated to determine $[\mathrm{F}]_{\max }$ (highest $\mathrm{F}$ concentration found in any collected AS sample over the 24hour period) as well as $F_{\text {total }}$ (the total amount of $F$ released from $\mathrm{FV}$ ).

\section{Statistical Analysis}

A one-way analysis of variance statistical method was used for data analysis. An overall significance of $(\alpha=0.05)$ was used. Pairwise comparisons between the groups were conducted using Tukey's method. Pearson correlation coefficients were calculated to investigate associations between the study variables.

\section{RESULTS}

Specimens treated with Nupro had the highest

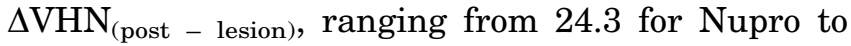
11.7 for Vella for the specimens receiving active treatment (Figure 2). No significant differences were found among FV; however, Nupro was the only FV significantly different from all placebo varnishes.

Specimens treated with Sparkle had the highest rehardening values following the second acid chal-

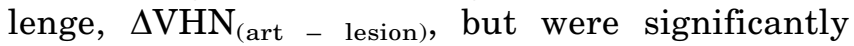
different only from those treated with Vella. All other FVs did not statistically affect lesion rehardening differently, but all FVs were significantly different from the placebo varnishes (Figure 3).

Fluoride release trends were somewhat similar for all tested FVs (Figure 4). The highest release for most FVs was within the first hour of testing, and then varnishes exhibited a steady decrease in fluoride release over time. MI released significantly more fluoride in the first hour than all the other test products $(9.71 \mu \mathrm{g} / \mathrm{mL})$. Enamel Pro came second $(5.44 \mu \mathrm{g} / \mathrm{mL})$, followed by Flor Opal (4.37 $\mu \mathrm{g} / \mathrm{mL}$ ); however, the latter two were not statistically different from each other. Flor Opal released in the first hour of the experiment approximately half that of MI. Butler White released significantly less fluoride $(0.12 \mu \mathrm{g} / \mathrm{mL})$ in the first hour than all other test products. Vella and Kolorz demonstrated atypical release behavior where peak release occurred at the five-hour collection point $(2.55 \mu \mathrm{g} /$ $\mathrm{mL})$.

For fluoride release total $\left(\mathrm{F}_{\text {total }}\right)$, MI released significantly more fluoride over the 24-hour incubation period of the experiment $\left(F_{\text {total }}=14.97 \mu \mathrm{g} / \mathrm{mL}\right)$ than all other FVs (Table 2). In addition, Enamel Pro and Flor Opal released more fluoride than the remaining FVs, except Nupro. Butler White released significantly less fluoride $\left(\mathrm{F}_{\text {total }}=0.50 \mu \mathrm{g} / \mathrm{mL}\right)$ than all other FVs.

The highest concentration of fluoride at any given time point was for MI $\left([\mathrm{F}]_{\max }=9.71\right)$, which was significantly higher than all FVs except for Enamel Pro $\left([\mathrm{F}]_{\max }=5.44\right)$, with Enamel Pro and Flor Opal having higher peak concentrations than all remaining FVs. Butler White exhibited the lowest peak concentration $\left([\mathrm{F}]_{\max }=0.17\right)$ and was significantly lower than all other FVs under study (Table 2).

Differences between FVs were less prominent for EFU than for $F_{\text {total }}$. PreviDent, Duraphat, and Vanish had significantly higher EFU than Cavity Shield, MI, Flor Opal, and Butler White but were not significantly different from the other FVs (Figure 5).

There were statistically significant but weak linear associations between $\Delta \mathrm{VHN}_{\text {(post }- \text { lesion) }}$ and $\mathrm{EFU}(r=0.36 ; p<0.0001), \Delta \mathrm{VHN}_{\text {(post }- \text { lesion) }}$ and $\mathrm{F}_{\text {total }}$ $(r=0.22 ; p=0.0011)$, and $\mathrm{F}_{\text {total }}$ and $\mathrm{EFU}(r=0.21$; $p=0.0022)$.

\section{DISCUSSION}

In this in vitro study, we aimed to investigate the efficacy of 14 commercially available FVs using the same outcome variables from one of our previous in vitro investigations. ${ }^{14}$ While the two studies investigated the effect of FVs on caries lesions, the study models were fundamentally different. The wide variation in performance of FV in our first experiment prompted the design of this in vitro study. We aspired to have a better understating of how different formulations affect the efficacy of FVs. Therefore, in this experiment, we aimed to investigate a larger variety of FVs in an attempt to have a range of products that represent different formulations. Also, in this study, FVs were left on the 


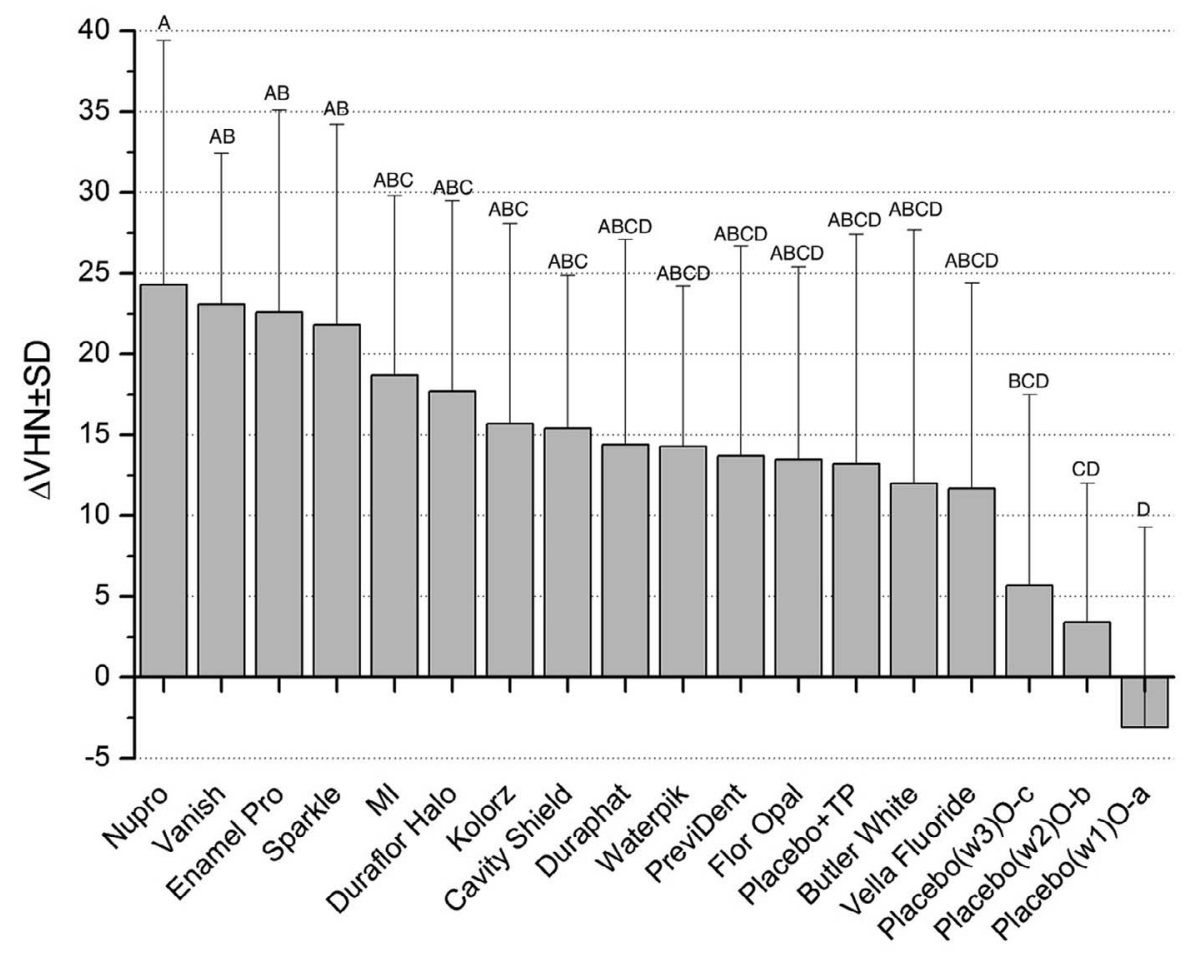

3
2

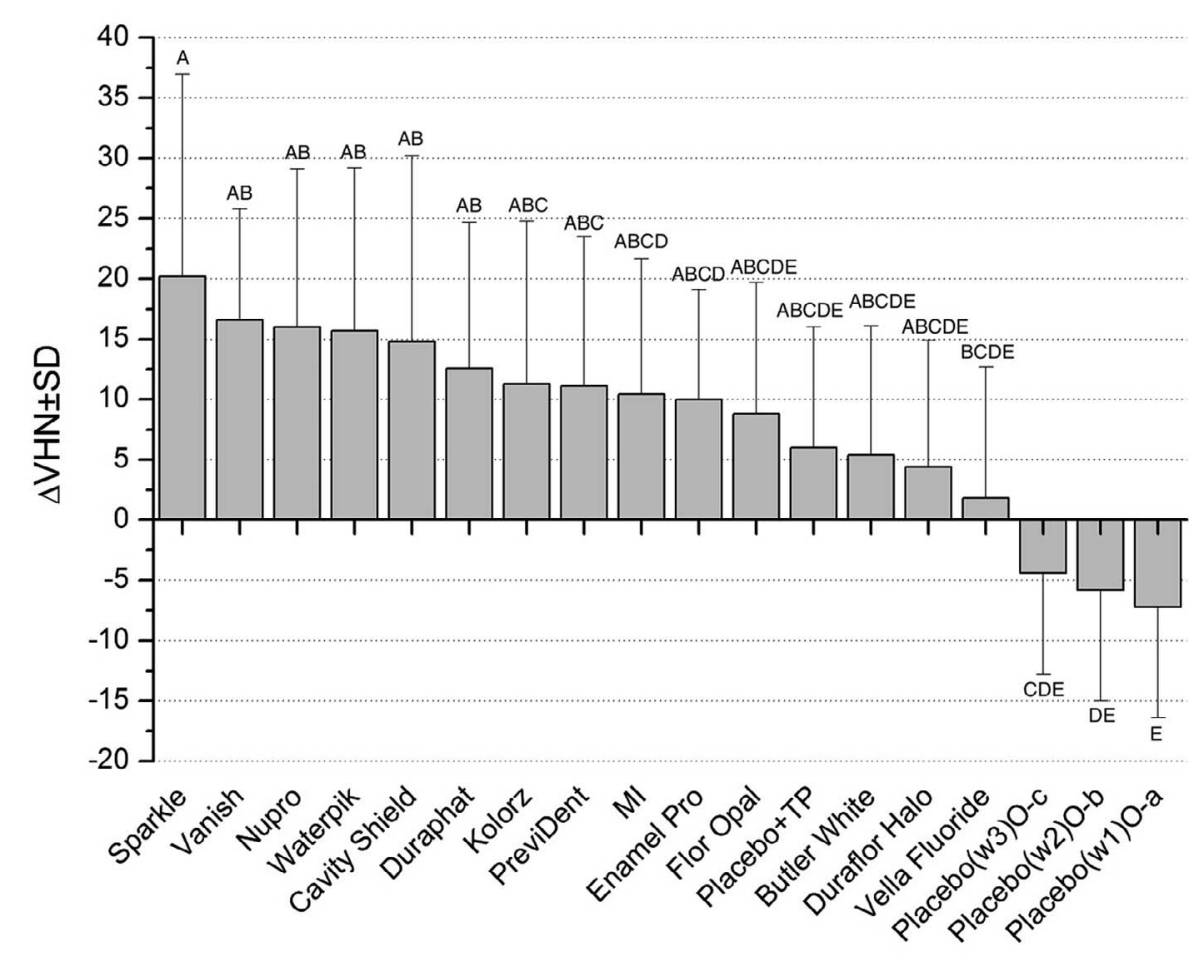

Figure 2. Mean change in surface microhardness ( $\Delta \mathrm{VHN}_{\text {(post - lesion) }) \text { as }}$ a function of fluoride varnish treatment. Significant differences between varnishes are highlighted by different letters. Error bars denote standard deviations.

Figure 3. Mean change in surface microhardness ( $\left.\Delta \mathrm{VHN}_{\text {(art - lesion) }}\right)$ as a function of fluoride varnish treatment. Significant differences between varnishes are highlighted by different letters. Error bars denote standard deviations. 


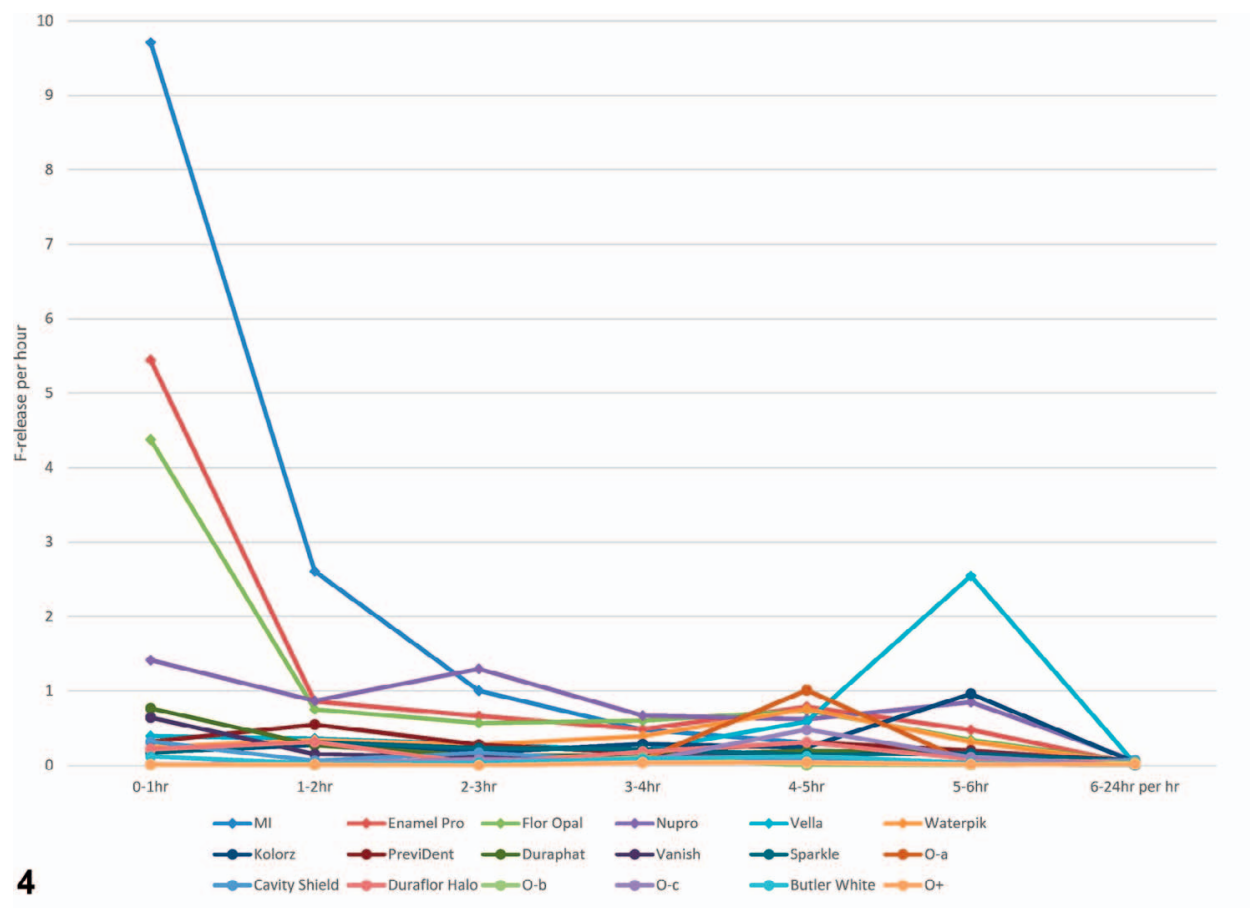

Figure 4. Mean hourly fluoride release from fluoride varnishes into saliva.

Figure 5. Enamel fluoride uptake (EFU) as a function of fluoride varnish treatment. Different letters highlight significant differences between varnishes. Error bars denote standard deviations.

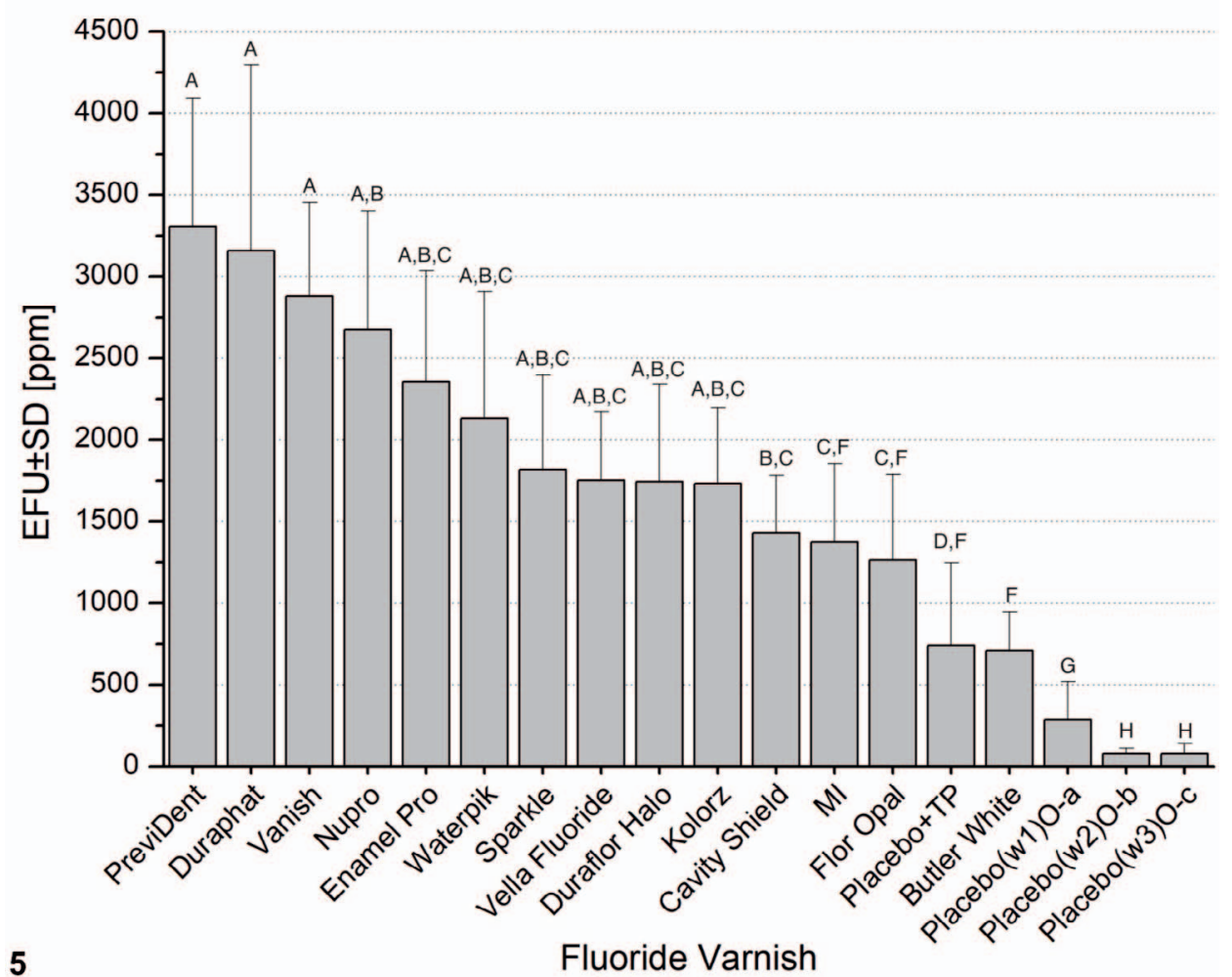

specimens for a prolonged period of 24 hours versus the six-hour period of the first study to better simulate the clinical situation.

This in vitro experiment's results for $\Delta \mathrm{VHN}_{\text {(post - }}$ lesion) and for $\Delta \mathrm{VHN}_{\text {(art - lesion) }}$ were unanticipated. As there are numerical differences in lesion rehar- dening values from different FVs, most of the FVs did not statistically differ from each other. Unfortunately, it is hard to thoroughly interpret the results because of the lack of detailed information on different FV formulations. However, there seems to be a superior effect on lesion rehardening when 


\begin{tabular}{|c|c|c|}
\hline Fluoride Varnish & Cumulative Fluoride Release $[\mu \mathrm{g} / \mathrm{mL}]$, Mean (SD) & Peak Fluoride Concentration $[\mu \mathrm{g} / \mathrm{mL}]$, Mean (SD) \\
\hline $\mathrm{Ml}$ & $14.97(2.38)$ A & $9.71(1.40)$ A \\
\hline Flor Opal & $8.20(1.91) \mathrm{B}$ & $4.37(0.85)$ B \\
\hline Nupro & $6.96(1.26)$ в, С & $1.69(0.43) \mathrm{C}, \mathrm{D}$ \\
\hline Vella & $4.91(2.02) \mathrm{C}, \mathrm{D}$ & $2.57(2.17) \mathrm{c}$ \\
\hline Kolorz & $2.91(0.46) \mathrm{D}, \mathrm{E}$ & $1.00(0.11) \mathrm{C}, \mathrm{D}, \mathrm{E}$ \\
\hline PreviDent & $2.82(0.59) \mathrm{E}, \mathrm{F}$ & $0.98(0.09) \mathrm{C}, \mathrm{D}, \mathrm{E}$ \\
\hline Duraphat & $2.64(0.53) \mathrm{E}, \mathrm{F}$ & $0.97(0.12) \mathrm{C}, \mathrm{D}, \mathrm{E}$ \\
\hline Vanish & $2.63(0.53) \mathrm{E}, \mathrm{F}$ & $1.28(0.09) \mathrm{C}, \mathrm{D}, \mathrm{E}$ \\
\hline Sparkle & $2.19(0.47) \mathrm{E}, \mathrm{F}$ & $0.95(0.15) \mathrm{C}, \mathrm{D}, \mathrm{E}$ \\
\hline$O-b$ & $0.85(0.17) \mathrm{G}$ & $0.71(0.16) \mathrm{E}$ \\
\hline O-c & $0.81(0.55) \mathrm{G}, \mathrm{H}, \mathrm{I}$ & $0.55(0.51) \mathrm{F}$ \\
\hline Butler White & $0.50(0.15) \mathrm{H,I}$ & $0.17(0.09) \mathrm{H}$ \\
\hline $\mathrm{O}+$ & $0.49(0.16)$ । & $0.37(0.16) \mathrm{G}$ \\
\hline
\end{tabular}

calcium- and phosphate-containing ingredients are added to the formulation. This is in agreement with our previous in vitro study. ${ }^{14}$ This may be attributed to the abundant amount of calcium and phosphate from these formulations that is readily available to interact with the teeth. It is important to note that the enhanced rehardening effect from calcium- and phosphate-containing ingredients did not withstand the second acid challenge. This is in contrast to a recent systematic review that suggested a possible long-term effect (>three months) of casein phosphopeptide-ACP (CPP-ACP) complexes on early caries lesions; however, the results cannot be extrapolated as the review investigated CPP-ACP alone because of insufficient evidence on the complex's synergistic effect with fluoride. ${ }^{15}$ Another noteworthy finding is that lesions treated with Vanish, an FV-containing functionalized tricalcium phosphate (fTCP), had a higher $\Delta \mathrm{VHN}_{\text {(post - lesion) }}$ than those treated with FVs containing ACP and CPP-ACP. This may be due to the protective effect of functionalization of the TCP molecule that prevents premature interaction between calcium and fluoride and aids in remineralization in a manner similar to that of fluoride. ${ }^{16}$

The fluoride release data in our experiment are in agreement with our previously mentioned in-house experiment and the 2014 study by Cochrane and others. ${ }^{12,13}$ Calcium-containing FVs, with the excep- tion of fTCP-containing varnishes, were able to release significantly higher levels of fluoride into saliva. This suggests a synergistic effect of adding casein complexes to FVs on fluoride release and may be explained by the bioavailable nature of ACP and CPP-ACP compared with the less-soluble fTCP. ${ }^{16-18}$

EFU data from this study extend our results from our aforementioned in vitro investigation. Once again, a CPP-ACP-containing varnish delivered less fluoride into caries lesions. This may be a result of the negative impact that inorganic phosphate released from CPP-ACP varnishes has on the formation of $\mathrm{CaF}_{2}$, thus reducing bioavailable fluoride. Vanish, an FV-containing fTCP, delivered more fluoride into lesions compared with CPP-ACP and ACP-containing FV despite its low fluoride release into saliva. This is in agreement with a study that compared EFU from two varnishes, one containing fTCP and the other containing CPP-ACP. ${ }^{17}$ Also, another study compared EFU with and without fTCP. It was found that lesions exposed to fluoride in conjunction with fTCP had significantly higher fluoride uptake than those exposed to fluoride alone, and the effect of fTCP was dose dependent. The mechanism of action of fTCP on enhancing lesion uptake of fluoride is not fully understood but may be attributed to the ability of fTCP to promote fluoridebased nucleation. ${ }^{16}$ 
Contrary to our findings in our preceding in vitro study, we were able to demonstrate a significant but weak linear association between $\Delta \mathrm{VHN}_{\text {(post - lesion) }}$ and EFU. To the best of our knowledge, this is the first time an association was established between $\triangle \mathrm{VHN}$ and EFU in FV research. We are also in agreement with another study in the lack of meaningful correlation between fluoride release and EFU. ${ }^{19}$ Based on our findings, we believe that fluoride release cannot be used as a predictive measure for EFU, and therefore, one should be careful in relating fluoride release to FV efficacy.

While our findings are in agreement with some studies and in disagreement with others, it is important to remember that all $\mathrm{FV}$ in vitro models have their limitations to the extent that the in vivo situation cannot be mimicked because of the complexity of in vivo caries. With FV in particular, there is the issue of infrequent applications, which makes modeling much harder. In our model, we tried to mimic the in vivo application more closely. Most other models measure only the direct effect of an FV treatment while ignoring the effect of topical fluorides applied between FV applications. This makes our study more clinically relevant but also more challenging, as there are competing effects of the topical fluorides.

\section{CONCLUSION}

The results of this study demonstrated variation in FV efficacy in in vitro conditions, most likely because of differences in composition. Further investigations are needed to develop models that will help us understand FV behavior.

\section{Acknowledgement}

The authors would like to thank the staff at Oral Health Research Institute (Indianapolis, Indiana) for their help throughout the project.

\section{Conflict of Interest}

The authors of this article certify that they have no proprietary, financial, or other personal interest of any nature or kind in any product, service, and/or company that is presented in this article.

\section{(Accepted 23 February 2019)}

\section{REFERENCES}

1. Strohmenger L \& Brambilla E (2001) The use of fluoride varnishes in the prevention of dental caries: a short review Oral Disease 7(2) 71-80.
2. Helfenstein U \& Steiner M (1994) Fluoride varnishes (Duraphat): a meta-analysis Community Dentistry and Oral Epidemiology 22(1) 1-5.

3. Petersson LG (1993) Fluoride mouthrinses and fluoride varnishes Caries Research 27(Supplement 1) 35-42.

4. Clark DC (1982) A review on fluoride varnishes: an alternative topical fluoride treatment Community Dentistry and Oral Epidemiology 10(3) 117-123.

5. Carvalho DM, Salazar M, Oliveira BH, \& Coutinho ES (2010) Fluoride varnishes and decrease in caries incidence in preschool children: a systematic review Revista Brasileira de Epidemiologia 13(1) 139-149.

6. Featherstone JD (2009) Remineralization, the natural caries repair process-the need for new approaches Advances in Dental Research 21(1) 4-7, 10.1177/ 0895937409335590.

7. Ripa LW (1987) Topical fluorides: a discussion of risks and benefits Journal of Dental Research 66(5) 1079-1083.

8. Beltran-Aguilar ED, Goldstein JW, \& Lockwood SA (2000) Fluoride varnishes: a review of their clinical use, cariostatic mechanism, efficacy and safety Journal of the American Dental Association 131(5) 589-596.

9. Buzalaf MA, Hannas AR, Magalhaes AC, Rios D, Honorio HM, \& Delbem AC (2010) pH-cycling models for in vitro evaluation of the efficacy of fluoridated dentifrices for caries control: strengths and limitations Journal of Applied Oral Science 18(4) 316-334.

10. White DJ (1992) The comparative sensitivity of intraoral, in vitro, and animal models in the 'profile' evaluation of topical fluorides Journal of Dental Research 71(Spec No) 884-894.

11. Zero DT (1995) In situ caries models Advanced Dental Research 9(3) 214-230.

12. Cochrane NJ, Shen P, Yuan Y, \& Reynolds EC (2014) Ion release from calcium and fluoride containing dental varnishes Australian Dental Journal 59(1) 100-105, 10. 1111/adj.12144.

13. Jablonowski BL, Bartoloni JA, Hensley DM, \& Vandewalle KS (2012) Fluoride release from newly marketed fluoride varnishes Quintessence International 43(3) 221-228.

14. Al Dehailan L, Martinez-Mier EA, \& Lippert F (2015) The effect of fluoride varnishes on caries lesions: an in vitro investigation Clinical Oral Investigations 20(7) 1655-1662, 10.1007/s00784-015-1648-4.

15. Li J, Xie X, Wang Y, Yin W, Antoun JS, Farella M, \& Mei L (2014) Long-term remineralizing effect of casein phosphopeptide-amorphous calcium phosphate (CPPACP) on early caries lesions in vivo: a systematic review Journal of Dentistry 42(7) 769-777, 10.1016/j.jdent. 2014.03.015.

16. Karlinsey RL \& Pfarrer AM (2012) Fluoride plus functionalized beta-TCP: a promising combination for robust remineralization Advanced Dental Research 24(2) 48-52, 10.1177/0022034512449463. 
17. Schemehorn BR, Wood GD, McHale W, \& Winston AE (2011) Comparison of fluoride uptake into tooth enamel from two fluoride varnishes containing different calcium phosphate sources Journal of Clinical Dentistry 22(2) 51-54.

18. Karlinsey RL, Mackey AC, Stookey GK, \& Pfarrer AM (2009) In vitro assessments of experimental $\mathrm{NaF}$ dentifrices containing a prospective calcium phosphate technology Americal Journal of Dentistry 22(3) 180-184.

19. Bolis C, Hartli GP, \& Lendenmann U (2015) Fluoride varnishes-is there a correlation between fluoride release and deposition on enamel? Oral Health \& Preventive Dentistry 13(6) 545-556, 10.3290/j.ohpd. a34373. 